Convergences francophones $2.2(2015): 34-45$

http://mrujs.mtroyal.ca/index.php/cf/index

\title{
Alexandre Csoma de Kőrös, le traducteur-rhinocéros
}

\author{
Gilles Mossière \\ Mount Royal University
}

Dans le cadre de la journée d'étude du 30 avril 2015 sur la figure du traducteur/de la traductrice, nous avons présenté le traducteur hongrois Alexandre Csoma de Körös (1784-1842) en faisant écho à la notion polysémique de figure, celle-ci pouvant être prise dans le sens de visage, mais aussi comme un trope rassemblant des représentations métaphoriques ou symboliques. ${ }^{1}$ Alexandre Csoma de Körös est un personnage peu connu internationalement, mais c'est une figure historique incontournable de sa Hongrie natale pour avoir établi le premier dictionnaire tibétain-anglais et la première grammaire du tibétain en 1834. Ce faisant, il a créé le domaine de la tibétologie. Dans son pays, c'est un héros national, et nombre de rues, boulevards, écoles, etc. portent son nom. Il a aussi fait l'objet de plusieurs films. ${ }^{2}$ Des associations hongroises continuent d'entretenir la mémoire et l'œuvre de ce linguiste-traducteur en plaçant des plaques sur les lieux de son passage, en organisant des événements commémoratifs et en aidant à la rénovation de monastères himalayens dans lesquels il a séjourné. ${ }^{3}$

Dans ce contexte, notre article fait également écho aux propos du conférencier invité, le Pr. Lieven D'hulst, sur le rôle du traducteur en tant que médiateur culturel, et sur l'apport de la traduction à la compréhension de l'histoire, en particulier à la compréhension des pratiques culturelles historiques, dont la langue et la religion font évidemment partie (D'hulst Essai 21).

Pour la notion de traducteur-rhinocéros, nous nous sommes inspiré de la biographie de Sylvain Jouty, publiée en 2007, qu'il a intitulée Celui qui vivait comme un rhinocéros: Alexandre Csoma de Körös (1784-1842), le vagabond de l'Himalaya. Pour cet article, nous commencerons par une présentation physique de Sándor et des diverses représentations qui ont été faites de lui, puis nous reviendrons brièvement sur ses voyages et lieux et conditions de résidence. Comme Sylvain Jouty ne jouit pas non plus d'une réputation internationale, nous ferons également une brève présentation de cet auteur. Nous nous intéresserons ensuite à l'association des termes « traducteur » et « rhinocéros » pour en dégager les tenants et aboutissants.

\section{Figures d'Alexandre Csoma de Kőrös}

\footnotetext{
${ }^{1}$ En cela, nous suivons, entre autres, la notion de «polysémie de figure» qu’Yves Gambier a posée en introduction de son article «Le traducteur défiguré » dans La Figure du traducteur (sous la direction d'Elżbieta Skibińska).

${ }^{2}$ Entre autres, A Guest of Life de Tibor Szemző (2006) et Zangla - Path of Csoma de Zoltán Bonta (2008). Des extraits du documentaire de Péter Molnár, The Testament of Alexander Csoma de

Körös, sont disponibles sur YouTube mais uniquement en hongrois:

<www.youtube.com/watch?v=YnplugbxHc4>.

${ }^{3}$ Voir Csoma ss Room Project, August 2012, Zangla, Ladakh, India, documentaire de la fondation hongroise Csoma's Room sous-titré en anglais : www.youtube.com/watch?v=PspXCz8dpXc.
} 
Alexandre Csoma de Körös n'est pas un aristocrate comme la particule pourrait le faire croire. Körösi Csoma Sándor, de son nom hongrois, est issu d'une famille de modestes agriculteurs. Körös est le nom du village où il est né, à l'extrémité orientale de la Transylvanie (village désormais situé en Roumanie), Csoma est son nom de famille, et Sándor est l'équivalent hongrois d'Alexandre. ${ }^{4}$ Sylvain Jouty en donne une description assez banale : «taille médiocre, visage rond, yeux noirs jaunâtres, nez droit, cheveux noirs, moustache rasée » (89), avec « ses traits un peu lourds, il pouvait tout à fait passer pour un honnête Arménien » (101). ${ }^{5}$ Cette description est toutefois immédiatement problématique puis qu'elle repose sur le seul et unique portrait qui existe de Sándor (fig.1).

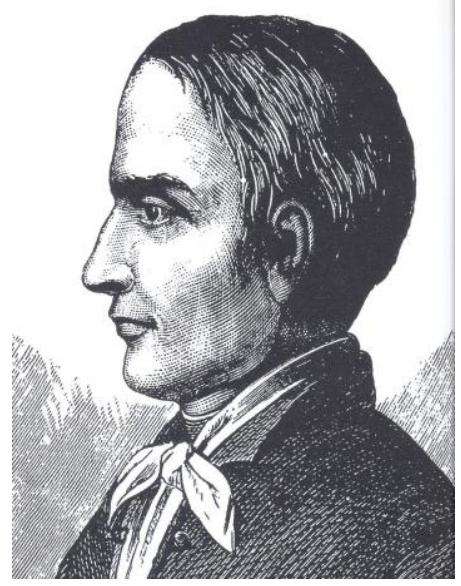

Fig. 1 Portrait volé

Connu sous le nom de «portrait volé », il a été exécuté par le peintre hongrois August Schoefft à Calcutta en 1840, donc deux ans avant la mort de Sándor. Il est appelé «volé » parce que Sándor avait refusé catégoriquement que Schoefft fasse un portrait de lui, mais comme le peintre hongrois avait un talent de dessinateur hors pair, il ajoutait un ou deux traits à son esquisse dès que son compatriote avait le dos tourné. Sándor avait refusé de poser, arguant que «ce n'était pas son visage qui devait passer à la postérité, mais son œuvre » (314). Dans ce contexte, la première figure du traducteur est donc de type «classique», celle du « traducteur invisible», uniquement au service des

textes source et d'arrivée. Le souhait d'anonymat de Sándor n'a pas non plus été reconnu après sa mort: il existe un certain nombre de représentations du traducteur linguiste, mais nous nous limiterons aux trois plus importantes: le buste créé par Holló Barnabás et présenté par l'Académie des sciences de Hongrie à la Société asiatique du Bengale (fig.2), la statue de Géza Csorba, qui est placée sur le mémorial de l'université bouddhiste de Tokyo, et un portrait au centre d'un tanka tibétain. ${ }^{6} \mathrm{Si}$ ces images existent, en plus de sa notoriété de premier traducteur tibétain-anglais, c'est que Sándor

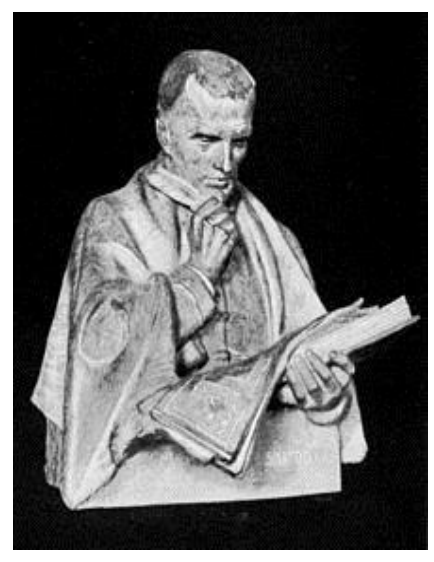

Fig.2 Buste de Barnabás

\footnotetext{
${ }^{4}$ Dans cet article, à la suite de $\mathrm{S}$. Jouty, nous abrégerons ce nom en n'utilisant que le prénom hongrois Sándor.

${ }^{5}$ Cette caractérisation pouvait donc lui permettre de rester relativement incognito dans les contrées orientales qu'il a traversées comme nous le verrons plus avant.

${ }^{6}$ Nous avons inclus les deux images qui sont libres de droit d'auteur (respectivement, pour le «portrait volé » et pour le buste de Barnabás), mais ne pouvons que fournir les liens pour la statue de Czorba : et pour le tanka tibétain.
} 
a été reconnu comme «bodhisattva » dès le 22 février 1933, «bodhisattva » étant une notion bouddhiste correspondant à la figure d'un sage qui, ayant atteint l'illumination, renonce à la libération du cycle des réincarnations pour aider les autres à parvenir à ce même état de conscience. A notre connaissance, il est le seul Européen à qui cet honneur a été décerné.

Ce qu'il est intéressant de remarquer dans ces représentations, c'est qu'aucune ne correspond au modèle du «portrait volé ». En effet, dans le buste de Barnabás, Sándor a les cheveux courts et un visage sévère très anguleux; dans la statue de Czorba, il est représenté assis en position de lotus, les cheveux rasés avec un visage de type plutôt oriental; dans le tanka tibétain, s'il a bien les cheveux milongs et noirs, la peau de son visage est totalement blanche. Il semble donc que tous ces artistes aient cherché à aller au-delà de l'apparence physique de Sándor - telle que représentée dans «le portrait volé »-et à mettre l'accent sur l'ascèse centrale à son entreprise (comme nous le préciserons), et ce faisant à participer à la mythification de ce personnage. Pour comprendre ce phénomène, nous rappellerons brièvement le trajet qui a amené Sándor à la connaissance, dans le sens fort du terme, du tibétain et des notions bouddhistes. Afin de mieux comprendre ce qui lui a valu cette notoriété non désirée, nous donnerons quelques détails sur l'envergure de son/ses entreprise(s). La carte ci-après donne une bonne idée de son périple, et laisse imaginer les difficultés de toutes sortes auxquelles il dut faire face.

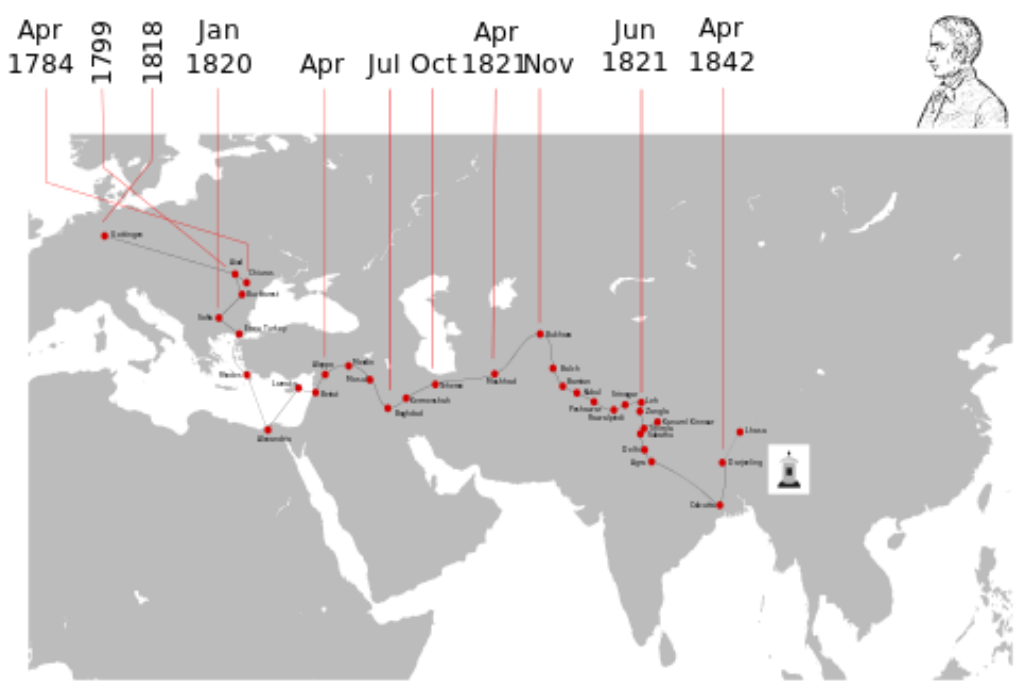

Fig. 3« Travel route and timeline of Sándor Körösi Csoma»

Sándor avait quitté sa Hongrie natale en 1819, à l'âge de 36 ans, à pied, muni de très peu d'argent et d'un simple baluchon, pour retrouver les racines de la langue hongroise, qu'on pensait descendre de celle des Huns, dans les montagnes du 
Turkestan et de la Mongolie. ${ }^{7}$ Ne pouvant dépasser Boukhara dans le sud du Turkestan ni ensuite traverser la chaîne du Karakoram à partir du Ladakh, Sándor dut se résoudre à retarder son projet, et à accepter celui du Superintendant des haras des Indes William Moorcroft d'apprendre le tibétain et d'établir un dictionnaire tibétain-anglais, langue totalement mystérieuse à l'époque.

Avant d'être un traducteur-passeur dans le sens traditionnel de «disséminateur » du terme, Sándor a donc été un grand voyageur, et sa notoriété est le résultat totalement inopiné d'un trajet qui avait un tout autre objectif. ${ }^{8} \mathrm{Ce}$ périple renferme aussi une ironie assez remarquable: en effet, depuis 1764, l'impératrice autrichienne Marie-Thérèse avait décidé que les habitants de la Transylvanie natale de Sándor feraient office de garde-frontières pour protéger l'est de l'empire, et Sándor a passé sa vie à se heurter à tous les garde-frontières de l'Orient...

Pour instruire Sándor dans la langue tibétaine, Moorcroft avait trouvé un lama, Sangye Phuntsog, et une forteresse semi-désaffectée, celle de Zangla, au Zanskar, région très reculée du Ladakh pour ne pas provoquer la curiosité intempestive des administrations locales et chinoises. Sándor séjourna dans ce lieu situé à 3900 mètres d'altitude de juin 1823 à octobre 1824, dans des conditions d'une extrême dureté sur lesquelles nous reviendrons. Sándor passa ensuite du temps dans les monastères de Zongkhul (qui, contrairement à Zangla possédait une bibliothèque), de Phuktal (situé à $4200 \mathrm{~m}$ d'altitude, en 1825 et 1826) et de Kanum (situé dans une zone plus clémente à $2800 \mathrm{~m}$, de 1827 à 1830). ${ }^{9}$ Ayant terminé ses études, il se dirigea alors vers la Société asiatique du Bengale à Calcutta où il rédigea son dictionnaire et sa grammaire, qui furent publiés en 1834.

Avant Sylvain Jouty, un certain nombre d'auteurs se sont intéressés à Sándor et ont reconstitué son parcours et son existence sur la base des maigres renseignements qu'il a consignés dans ses rapports à la Société asiatique, ou que d'autres ont inclus dans leurs récits de voyages. ${ }^{10}$ Son premier biographe fut Theodore Duka, un chirurgien hongrois qui a travaillé en Inde (1885); Bernard Le Calloc'h est un linguiste français qui a publié plusieurs articles sur Sándor et qui

\footnotetext{
${ }^{7}$ L'idée d'identification à cette descendance des Huns correspondait, à cette époque, à une poussée nationaliste contre l'empire autrichien. Il est désormais accepté que cela n'est nullement le cas, et que le hongrois fait partie du groupe des langues finno-ougriennes.

${ }^{8}$ Dans Translation Studies, Susan Bassnett, suivant Michael Cronin (Across the Lines: Travel, Language, Translation. Cork: Cork University Press, 2000), rappelle que le traducteur est aussi un voyageur, engagé dans un trajet d'une source à une autre, et que ce passage engage des considérations spatiales et temporelles (1-2). Le cas de Sándor, comme nous l'avons vu et continuerons de le voir dans cet article, dépasse largement le cadre du «traducteur-voyageur » envisagé par Basnett.

${ }^{9}$ Pour les raisons de droits d'auteur signalées plus haut, nous ne pouvons donner que les lienscomme références.

${ }^{10}$ Personnage hors du commun, Sándor a marqué ceux qui l'ont rencontré, entre autres, William Moorcroft et August Schoefft, évidemment, mais aussi le géologue britannique James Gerard et le naturaliste français Victor Jacquemond qui ont laissé de brèves évocations de Sándor dans leurs écrits.
} 
jouit d'une grande réputation pour cela en Hongrie ; ${ }^{11}$ et Edward Fox a écrit The Hungarian who walked to Heaven en 2001. ${ }^{12}$ Au Canada, Judith GalánthaHermann a publié plusieurs notices explicatives sur Sándor dans Lectures and Papers in Hungarian Studies. Une chose est cependant tout à fait évidente : aucun de ces textes ne possède l'ampleur et les détails - le souffle - de la biographie de Jouty. Cette dernière regorge de dates et de détails géographiques et biographiques, dont beaucoup se rapportent aux contextes historique et géopolitique de l'époque, mais nous n'avons pas été surpris de voir que Jouty avait ajouté, sur son site Internet, la mention «biographie romancée » au titre de son livre - mention qui ne figure pas dans le livre publié par Fayard. En effet, une question évidente se pose : comment faire le portrait d'un traducteur disparu depuis longtemps, et ce, de manière suffisamment réaliste pour qu'il soit crédible? Jouty n'est pas le premier à s'être frotté à ce problème, et nous évoquerons les travaux de la Canadienne Agnes Whitfield plus avant. Dans le cas de Sándor, l'immense difficulté vient du manque flagrant de traces écrites sur son processus de travail. Comment Jouty s'y est-il donc pris pour en restituer la teneur, c'est ce que nous verrons après avoir fait une brève présentation de cet auteur. Cela nous semble nécessaire puisque Jouty n'est pas non plus un auteur reconnu par le grand public bien qu'il ait publié de nombreux ouvrages, dont les romans L'Odeur de l'altitude (1999), Voyages aux pays évanouis (2000) et Le roman du Cervin (2003). ${ }^{13}$

\section{Brève présentation de Sylvain Jouty}

Né le 30 juin 1949 à Paris, c'est en 1955 qu'il fait ses débuts d'escalade à Fontainebleau et parvient au sommet de son premier sommet de $3000 \mathrm{~m}$. Cinq ans plus tard, c'est son premier 4000, le Bishorn (4 $153 \mathrm{~m})$ en famille, et l'année suivante sa première ascension du mont Blanc avec son père. Dès 1964 (à l'âge de quinze ans), il réalise ses premières ascensions sans parents ni guide, dont des voies classiques comme la traversée Charmoz-Grépon et la voie Rébuffat à l'aiguille du Midi. Ce bref aperçu indique que Jouty est quelqu'un d'assez peu ordinaire, ce qu'il a exprimé dans un message électronique au style laconique en adéquation avec son contenu: «Je comprends que je suis un peu différent, ce qui me culpabilisera longtemps: mon proviseur avait raison, je manque de motivation, je suis un dilettante-né - jusqu'en littérature, toujours un peu ailleurs, jamais complètement impliqué... Encore aujourd'hui j'ai parfois l'impression de n'être pas tout à fait concerné par ce qui m'advient. Bon». ${ }^{14}$

\footnotetext{
${ }^{11}$ Voir, entre autres, Bernard Le Calloc'h, Alexandre Csoma de Körös (1985). Le gouvernement hongrois lui a décerné la Croix d'or de l'ordre du Mérite pour l'ensemble de son œuvre.

${ }^{12}$ Réédité en 2006, ce petit livre de 96 pages reprend les éléments présentés par Duka. Il en est de même de même pour l'article du hongrois Ernest Hetenyi «Alexander Csoma de Körös the Hungarian Bodhisattva », et pour le livre Hermit-hero from Hungary de Hirendra Nath Mukerjee.

${ }^{13}$ Sans doute le plus connu, L'Odeur de l'altitude a obtenu le grand prix du livre de montagne en 1999, puis été publié dans la collection «Livre de Poche » en 2003, et été traduit en espagnol en 2001 et en russe en 2006. Pour complément d'information, voir www.jouty.com.

${ }^{14}$ Message électronique du 2 avril 2015.
} 
En 1969, il fait des études de chinois à l'université Dauphine à Paris, et y découvre le taoïsme - «toujours en dilettante, bien sûr ». Les années 1983-1998 correspondent à une période relativement plus sérieuse de son existence puisqu'il sera rédacteur de la revue Alpinisme et Randonnée. Il est administrateur et rapporteur général de la Société des gens de lettres de France depuis 2001, et directeur des collections « Retour à la montagne » et « Destins de montagne » aux éditions Hoëbeke (Paris). En ce qui concerne sa production littéraire, il est associé au mouvement de la « Nouvelle Fiction », comme l'indique un article publié dans le numéro de novembre 2000 du Magazine littéraire. ${ }^{15}$

L'intérêt de Jouty pour Alexandre Csoma de Körös date de 1989, année à laquelle il fait un voyage au Bhoutan et au Sikkim et publié un article sur lui dans la revue Alpinisme et Randonnée. L'idée d'écrire une biographie complète du traducteur-linguiste hongrois ne s'est cristallisée qu'au début des années 2000, après une panne de chauffage en plein milieu de l'hiver dans la maison qu'il habite dans les montagnes au-dessus de Buis-Les-Baronnies (Drôme), panne de chauffage aux désagréments tout relatifs, mais qui lui a cependant rappelé les conditions de travail de Sándor dans les montagnes du Zanskar.

\section{Le traducteur-rhinocéros}

En termes de symbolisme, le « rhinocéros » a plusieurs facettes, mais Jouty n'en retient qu'une: n'avoir qu'un seul objectif et y consacrer toute son attention. Dans sa biographie de Sándor, le « rhinocéros » est mentionné pour la première fois à la page 52, faisant écho aux paysages montagneux du Zanskar et à la quête infinie de sens dans laquelle est engagée l'humanité: "Derrière une crête, toujours une autre crête. Derrière une question toujours une autre question. Autrement dit nul n'en viendra jamais à bout, sinon la chaîne entière de l'humanité. Il fallait donc se contenter d'un seul but, et ne jamais s'en détourner, comme un rhinocéros qui charge ». (C'est nous qui soulignons.)

Le thème de l'opiniâtreté qui se dégage de l'extrait ci-dessus est largement développé tout au long du livre (pages 58, 60, 61, 65, 67, 85, 86, 124, etc.), mais la deuxième mention spécifique du rhinocéros n'apparaît que 190 pages après la première - nous y reviendrons bientôt.

Comme nous l'avons mentionné, après trois années et demie de voyage dans les conditions difficiles du début du XIX ${ }^{\mathrm{e}}$ siècle, ${ }^{16}$ il fallut à Sándor dix ans d'études et de travail dans les conditions encore plus extrêmes du Zanskar pour mener à bien son entreprise de traduction (219, et al.). Ces difficultés n'étaient pas une surprise

\footnotetext{
${ }^{15}$ Francis Berthelot, «La Nouvelle Fiction », Magazine littéraire 392, 30-33. La « Nouvelle Fiction » se définit par une opposition au courant dominant du « réalisme » dans la littérature française contemporaine, et par une radicalisation du courant « imaginaire » hérité, entre autres, de Rabelais, Nerval, Cocteau, Vian, Pieyre de Mandiargues « pour revendiquer les valeurs extrêmes du discours fictionnel ». L'association de Jouty avec le mouvement de la " nouvelle fiction » s'applique beaucoup moins à sa biographie d'Alexandre Csoma de Körös qu'à ses romans, tels que L'Odeur de l'altitude.

${ }^{16} \mathrm{Il}$ commence son périple le 19 novembre 1819 et arrive au Zanskar le 26 juin 1823.
} 
pour Sándor: il s'y était préparé pendant plusieurs années en Hongrie dès l'âge de vingt ans (1804). Les descriptions fournies par Jouty sont très claires: « Il savait également que cela serait très dur ... Il devait donc s'habituer à supporter sans gêne des conditions encore plus difficiles. Il ne devait plus goûter le confort; c'était l'inconfort qui devait lui devenir confortable » (65).

Dormant sur des planches nues ou à même le sol, restant parfois plusieurs jours sans manger, «il apprenait systématiquement à refuser toute douceur, à décliner toute tentation; il s'agissait moins de pouvoir résister à celle-ci que de l'annihiler» (ibid.). Pour Jouty, Sándor s'approchait ainsi sans le savoir d'un concept bouddhiste inconnu en Europe, et qu'il allait être l'un des premiers à devoir traduire: "Extinction du désir, extinction des besoins, nirodhah, permettant la disparition de la souffrance, duhkha: c'est l'une des Quatre Nobles Vérités de l'Octuple sentier menant au nirvāna » (65-66).

Pour Sándor, la tâche, en effet, était immense : outre les conditions de vie d'une dureté extrême qu'il avait à supporter, il devait tenter de déchiffrer un vocabulaire inconnu, à l'écriture complexe, et une grammaire qui l'était tout autant, et qui exprimait des concepts et une pensée pour lesquels il n'avait aucun point de référence. Pour ce faire, il avait Sangye Phuntsog comme professeur, un lama érudit, mais qui ne parlait que le tibétain, et qui ne l'avait jamais enseigné à un étranger:

[le lama] procédait toujours de même, épelant les mots par une récitation psalmodiée en même temps qu'il traçait les syllabes. Cela paraissait un excellent procédé de mémorisation, et Sándor s'efforçait de répéter à sa suite, mais sans pouvoir saisir ni le sens des mots, ni celui des lettres, et encore moins des notions bien trop nébuleuses. Tout ça était trop compliqué. (225-6)

Pour donner une 'petite' idée de la complexité de cet apprentissage (et du sens de l'humour de Jouty), nous proposons la longue citation ci-dessous:

Après les trente consonnes, le lama passa aux voyelles, indiquées sur les consonnes par les naro, guigou et autres chyabkyou placés au-dessus ou au-dessous. Ensuite ça se compliquait. Il y avait les lettres suscrites ou souscrites, parfois abrégées. Les lettres inversées, utilisées pour transcrire les expressions sanskrites. Les tons, qui différaient selon que les lettres étaient masculines, féminines, sous-féminines, extrêmement féminines, communes ou neutres. Les préfixes et affixes simples ou doubles, parfois prononcés, parfois muets, parfois affectant le son de la lettre suivante ou de la précédente... Pour s'y retrouver, il était nécessaire d'épeler la syllabe entière dans un ordre bien précis, d'abord le préfixe, puis la lettre suscrite, la racine, la lettre souscrite, le signe-voyelle, les suffixes, en indiquant à la fois l'ordre, le rôle de la lettre et les modifications apportée aux sons. Le lama lui donna l'exemple du mot-syllabe bsgribs $\square$ 줙 $\square$ त, qui se prononce drib : ba wo; sa tang; ga, ratak, dra, guigou, dri; ba; sa: DRIB. Le mot signifiait « obscur » et en effet ce n’était pas clair. (226-27) 
Avant de pouvoir traduire quoi que ce soit, il fallait donc que Sándor apprenne le tibétain, mais pour ce faire, il devait d'abord apprendre le sanskrit...

Il a donc fallu à Sandor une obsession sans borne et une opiniâtreté à toute épreuve pour avancer dans le déchiffrage, puis dans l'apprentissage du tibétain, ce qui nous ramène à la métaphore du rhinocéros - et du traducteur-rhinocéros.

En ce qui concerne le rôle de Sándor en tant que traducteur-rhinocéros, la référence essentielle - c'est-à-dire, celle qui donne leur unité à tous les exemples d'opiniâtreté que nous avons mentionnés - est celle de la page 242, où Sándor déchiffre un texte tibétain, avec sa difficulté coutumière. Sangye Phuntsog lui donne les clés de ce passage:

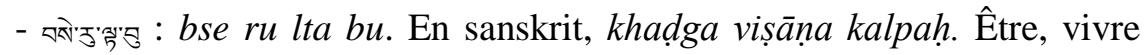
comme... un serou? Un khadga? Je ne sais pas ce que c'est.

- Un animal de l'Inde, plus gros qu'un yak, avec une grosse corne entre les yeux...

Rhinocéros, orrszarvú en hongrois : «celui avec une corne sur le nez ». Le rhinocéros était le symbole de celui qui se réalise dans la solitude. C'était aussi celui de l'obstination. On disait que rien ne détournait un rhinocéros de sa route, même pas les balles tirées pour l'arrêter.

Celui qui vit comme un rhinocéros (dans la solitude) : c'est ainsi que Sándor traduira en anglais la phrase numéro un du chapitre 45 de la Mahāvyutpatti. $(242)^{17}$

La citation ci-dessus effectue l'intégration complète de la métaphore du rhinocéros: figure de l'idéal bouddhiste, elle s'applique aussi parfaitement à la figure du traducteur, qui vivant cet idéal depuis des années est à même de le comprendre et de le traduire parfaitement. Si Alexandre Csoma de Körös a été reconnu comme «bodhisattva » le 22 février 1933 par les instances du bouddhisme au Japon, ce n'est pas seulement pour avoir ouvert les portes du domaine de la tibétologie, c'est aussi parce qu'elles considèrent qu'il avait réalisé cet idéal bouddhiste. $^{18}$

Agnes Whitfield a bien souligné la difficulté de faire le portrait d'un traducteur disparu en écrivant que cela tenait « davantage à la prestidigitation ».

\footnotetext{
${ }^{17}$ La Mahāvyutpatti est le premier dictionnaire bilingue d'importance à avoir été établi au monde. Compilé au Tibet à partir de la fin du VIII e siècle pour le roi Tridé Songtsen, il comprend des milliers de termes sanskrit-tibétain permettant de traduire les textes bouddhistes fondamentaux en tibétain. ${ }^{18}$ Bernard Le Calloc'h donne une définition simplifiée du terme « bodhisattva » : « titre prestigieux, comparable à ce que la sainteté est dans la tradition chrétienne ». Voir B. Le Calloc'h, « Alexandre Csoma de Körös, le bodhisattva hongrois » (1987). Le Calloc'h a publié de nombreux articles sur ce traducteur hongrois, dont « Alexandre Csoma de Körös, fondateur de la tibétologie (1784-1842) » (1985). Jouty reconnait brièvement sa dette par rapport aux travaux de Le Calloc'h : «Comme le remarque à peu près Bernard Le Calloc'h, qui avec son nom breton est un Français spécialiste du hongrois, de l'estonien et de Körösi Csoma Sándor [...]»(282).
} 
Pour cela, elle indiquait qu'il fallait marcher «sur la corde raide entre absence d'informations et formules creuses, entre suppositions et intuitions, entre renseignements filtrés et pistes nouvelles» 178). Comme nous l'avons remarqué, c'est à ce défi de "prestidigitation » que Jouty s'est attaqué pour écrire sa « biographie romancée » d'Alexandre Csoma de Körös. S'il s'est d'abord beaucoup renseigné - en lisant, entre autres, The Hungarian who walked to Heaven d'Edward Fox et les textes de Bernard Le Calloc'h,$-{ }^{19}$ c'est le terme d' « intuitions » mentionné par Whitfield qui a joué un rôle essentiel dans la reconstitution historique proposée par Jouty. Voici comment il décrit ce processus : «De manière plus générale, écrire ce genre de livre qui n'est pas totalement de fiction (au contraire je m'attache, comme dans Le Roman du Cervin, à suivre les faits avérés d'aussi près que possible), est en grande partie une histoire d'intuition » (c'est nous qui soulignons). ${ }^{20}$

Il semble que ce soit là, par-delà la simple recherche factuelle, que le travail de l'universitaire Whitfield et celui de l'écrivain Jouty se rejoignent. Celui-ci avait cependant la difficulté supplémentaire d'intégrer à sa biographie deux langues qu'il ne connaissait pas, le hongrois et le tibétain, ainsi que l'enseignement de cette dernière dans des conditions tout à fait hors normes:

J'ai donc tenté d'imaginer comment cela a pu se passer au vu des photos et dessins de la cellule du monastère de Zangla, d'une grammaire tibétaine, du lexique sanscrit-tibétain que Sandor eut en main (Mahāvyutpatti), des circonstances susdites, etc. Bref, cela ne s'est sans doute pas passé de la manière exacte dont je le raconte, mais celle-ci me paraît plausible dans ses grandes lignes. J'ai eu le même problème pour bien d'autres épisodes, bien entendu, et j'ai toujours tenté de faire le moins de « roman » possible (je veux dire par là ne pas inventer des éléments de toute pièce simplement pour enjoliver le récit, mais toujours tenter d'imaginer du mieux possible comment ça avait pu se passer). ${ }^{21}$

Au terme de cette brève évocation de l'œuvre d'Alexandre Csoma de Körös, nous laisserons les derniers mots à Sylvain Tesson, grand voyageur et écrivain moderne: «Csoma avait besoin de Sylvain Jouty. Le Hongrois est laconique, sa vie austère, ses recherches arides: il fallait qu'un marionnettiste au cœur large et à la plume rigoureuse s'impatronisât dans ce destin complexe, en démêlât les fils et

\footnotetext{
${ }^{19}$ Message électronique du 2 avril 2015. Jouty y déclare que le livre de Fox ne lui avait «pas beaucoup servi ». Il dit aussi : "J'ai lu bien sûr les textes de Le Calloc'h, mais je ne l'ai jamais rencontré. En revanche je lui ai envoyé mon livre, et je suis assez fier qu'il m'ait dit que c'était bien supérieur aux romans publiés en Hongrie sur ce personnage».

${ }^{20}$ Message électronique du 2 avril 2015.

${ }^{21}$ Message électronique du 19 juin 2015. Dans son message du 2 avril, Jouty avait indiqué : «J'irais même plus loin, pour bien écrire il faut "se laisser aller" à un état pas loin de la médiumnité. » Il est revenu sur cette déclaration dans son deuxième message : "Médiumnité est peut-être un peu fort, mais dans tous les cas je crois qu'il faut avant tout faire confiance à son intuition (tout en s'en méfiant). »
} 
Convergences francophones $2.2(2015): 34-45$

http://mrujs.mtroyal.ca/index.php/cf/index

fît jouer à son sujet la danse de l'aventure et de l'exploration sur les pistes hostiles de l'Asie intérieure » (n.p.). 


\section{Bibliographie}

\section{Illustrations}

Barnabás, Holló. «Le buste de Barnabas.» $<$ https://upload.wikimedia.org/wikipedia/commons/f/f1/CsomaDeKoros.jp $\mathrm{g}>$.

«anum.»:

<www.angelfire.com/trek/bameduniya/faroutpics/thumbnails/600x450/HP

«Phuktal.»: _from_then_till_2004.jpg>.

<www.gardenvisit.com/blog/wpcontent/uploads/2012/05/1582160281_23bc7c2cab_b.jpg>.

Schoefft, August. «Portrait volé.» $<$ https://upload.wikimedia.org/wikipedia/commons/c/cc/AlexanderCsoma KorosASB.jpg>.

«Statue de Géza Csorba. »<www.buddhatar.hu/sites/default/files/korosi_a_megvilagosult.jpg $>$.

«Tanka tibétain.» 〈www.korosi-emlekpark.hu/image/csoma/korositanka.jpg>. «Travel route and timeline of Sándor Körösi Csoma. » Carte composée par L. Shyamal (sous licence CC BY-SA 3.0 de Wikimedia Commons : $<$ https://commons.wikimedia.org/wiki/File:Travel_route_and_timeline_of _S\%C3\%A1nor_K\%C5\%91r\%C3\%B6si_Csoma.svg>.

«Zangla.»<http://csomasroom.kibu.hu/content_img/admin/admin1277541531>. «Zonkhul.»<www.journeymart.com/de/AttrationImages/zongkhul-monasteryzanskar.jpg >.

\section{Ouvrages}

Bassnett, Susan. Translation Studies (3 ${ }^{\mathrm{e}}$ éd.). London/New York: Routledge, 2004. Berthelot, Francis. «La nouvelle fiction. » Magazine littéraire $\mathrm{n}^{\circ} 392$ (novembre 2000), 30-33.

Bussières-Gallagher, Anne. Le traducteur fictif, personnage de la littérature québécoise (mémoire de maîtrise). Université de Sherbrooke, Savoirs UdeS (mai 2010). <http://savoirs.usherbrooke.ca/bitstream/handle/11143/2640/MR65660.pd $\mathrm{f}$ ? sequence $=1$ \&is Allowed $=\mathrm{y}>$.

D'hulst, Lieven. Essai d'histoire de la traduction. Paris: Classique Garnier, 2014. Duka, Theodore. Life and works of Alexander Csoma de Körös: a biography compiled chiefly from hitherto unpublished data; with a brief notice of each of his unpublished works and essays, as well as of his still extant manuscripts. Londres: Trübner, 1885.

Fox, Edward. The Hungarian who walked to Heaven. Londres : Faber \& Faber, 2006.

Galántha-Hermann, Judith. "The Intellectual Legacy of Alexander Csoma de Körös (1784 - 1842). » Lectures and Papers in Hungarian Studies, 1999. 
---. « New Discoveries about Alexander Csoma de Körös and the Buddhist Monasteries of

Northern India. » Lectures and Papers in Hungarian Studies, 2004.

Gambier, Yves. «Le traducteur défiguré. » La Figure du traducteur, Romanica Wratislavensia LIX (Elżbieta Skibińska, éd.). Wroclaw : 2012, 13-24.

Hetenyi, Ernest. "Alexander Csoma de Körös the Hungarian Bodhisattva. » <http://himalaya.socanth.cam.ac.uk/collections/journals/bot/pdf/bot_09_0 1_03.pdf $>$.1984, 34-41.

Jouty, Sylvain. Celui qui vivait comme un rhinocéros: Alexandre Csoma de Körös (1784-1842), le vagabond de l'Himalaya. Paris: Fayard, 2007 ;

---. "L'ermite du Zanskar: Alexandre Csoma de Körös », Alpinisme et Randonnée, 1986.

---. Message électronique à l'auteur, 2 avril 2015.

---. Message électronique à l'auteur, 19 juin 2015

Le Calloc'h, Bernard. Alexandre Csoma de Körös. Paris: La nouvelle revue tibétaine, 1985.

Mukerjee, Hirendra Nath, Hermit-hero from Hungary, Alexander Csoma de Koros, the great Tibetologist. New Delhi: Light \& Life Publishers, 1981.

Tesson, Sylvain. «Le sens hunnique. » Le Point 1817 (12 juillet 2007).

Whitfield, Agnes. "Méthode et pratique du portrait: Sur les traces des traducteurs. » La Figure du traducteur, Romanica Wratislavensia LIX (Elżbieta Skibińska, éd.). Wroclaw : 2012, 175-184.

---. «Émilie du Châtelet, traductrice d'Isaac Newton, ou la "traductionconfirmation." " Portraits de traductrices (Jean Delisle, éd.). Ottawa : Presses de l'université d'Ottawa, 2012, 87-115. 